\title{
The Efficacy of Bioabsorbable Mesh as an Internal Splint in Primary Septoplasty
}

\author{
Jee Nam Kim, Hyun Gon Choi, Soon Heum Kim, Hyung Jun Park, Dong Hyeok Shin, \\ Dong In Jo, Cheol Keun Kim, Ki Il Uhm \\ Department of Plastic and Reconstructive Surgery, Konkuk University School of Medicine, Seoul, Korea
}

\begin{abstract}
Nasal bone fractures are often accompanied by septal fractures or deformity. Posttraumatic nasal deformity is usually caused by septal fractures. Submucosal resection and septoplasty are commonly used surgical techniques for the correction of septal deviation. However, septal perforation or saddle nose deformity is a known complication of submucosal resection. Hence, we chose to perform septoplasty, which is a less invasive procedure, as the primary treatment for nasal bone fractures accompanied by septal fractures. During septoplasty, we used a bioabsorbable mesh as an internal splint. We used the endonasal approach and inserted the mesh bilaterally between the mucoperichondrial flap and the septal cartilage. The treatment outcomes were evaluated by computed tomography (CT) and the nasal obstruction symptom evaluation (NOSE) scale. The CT scans demonstrated a significant improvement in the septal deviation postoperatively. The symptomatic improvement rated by the NOSE scale was greater at 1 month and 6 months after surgery compared to the preoperative status. There were no cases of extrusion or infection of the implant. In cases of moderate or severe septal deviation without dislocation from the vomerine groove on the CT scan, our technique should be considered one of the treatments of choice.
\end{abstract}

Keywords Nasal septum / Nasal bone / Bioabsorbable implants
Correspondence: Hyun Gon Choi Department of Plastic and Reconstructive Surgery, Konkuk University School of Medicine, 120 Neungdong-ro, Gwangjin-gu, Seoul 143-729, Korea

Tel: $+82-2-2030-5235$

Fax: +82-2-2030-5249

E-mail: md58596@hanmail.net

This article was presented at the the 69th Annual Conference of the Korean Society of Plastic and Reconstructive Surgeons on November 11-13, 2011 in Seoul, Korea.

No potential conflict of interest relevant to this article was reported.

Received: 9 Mar $2012 \bullet$ Revised: 30 May $2012 \bullet$ Accepted: 11 Jun 2012

pISSN: 2234-6163 • elSSN: 2234-6171 • http://dx.doi.org/10.5999/aps.2012.39.5.561 • Arch Plast Surg 2012;39:561-564

\section{INTRODUCTION}

Because of the nose's central location and protruding position, the frequency of facial bone fractures is very high [1]. A nasal bone fracture can occur without a concurrent septal fracture, but in $20 \%$ of cases it can be accompanied by nasal septal damage [1]. If a fracture of the nasal septum is not treated appropriately, it can result in nasal deformation and nasal obstruction due to septal deviation. Since computed tomography (CT) has been utilized in clinics, enabling easy detection of nasal septal fractures, the importance of nasal septal correction has increased
$[1,2]$. The surgical technique used could be closed or open reduction depending on the degree of damage to the nasal septum; however, there are reports suggesting that aggressive procedures such as septoplasty or submucosal resection could be helpful in the treatment of septal fractures [1-3].

The parts of the nasal septum that are damaged in a nasal bone fracture are generally the perpendicular plate of the ethmoid bone or the quadrangular cartilage [2]. The perpendicular plate of the ethmoid bone supports the triangular structure that indicates the height and width of the nasal bone and the quadrangular cartilage forms the inferior pole of the nasal bone so it performs an impor- 
tant function of maintaining the shape of the nose and both the nasal airways. However, submucosal resection that is frequently performed in nasal septal fractures can give rise to complications such as septal perforation or saddle nose deformity.

Hence, we arrived at the conclusion that it is better not to perform submucosal resection through open reduction in a patient with a nasal bone fracture accompanied by a nasal septal fracture. Since the nasal cartilage tends to return to its original state before the damage, the authors invented a technique to maintain the reduction of the nasal septum for as long as possible after the operation has been performed [1]. Hence, the idea of application of an internal splint which can support and maintain the fractured nasal septum in an upright position was conceived. The technique consists of inserting a bioabsorbable mesh that is used frequently in facial bone fractures into the submucosa, and it yielded satisfactory results both in terms of aesthetic beauty and functionality.

\section{IDEA}

Between October 2010 and May 2011, a retrospective study was conducted on 20 patients who had been hospitalized at the authors' hospital with the chief complaint of a nasal bone fracture accompanied by a septal fracture. The age distribution of the patients was from 17 to 25 years.

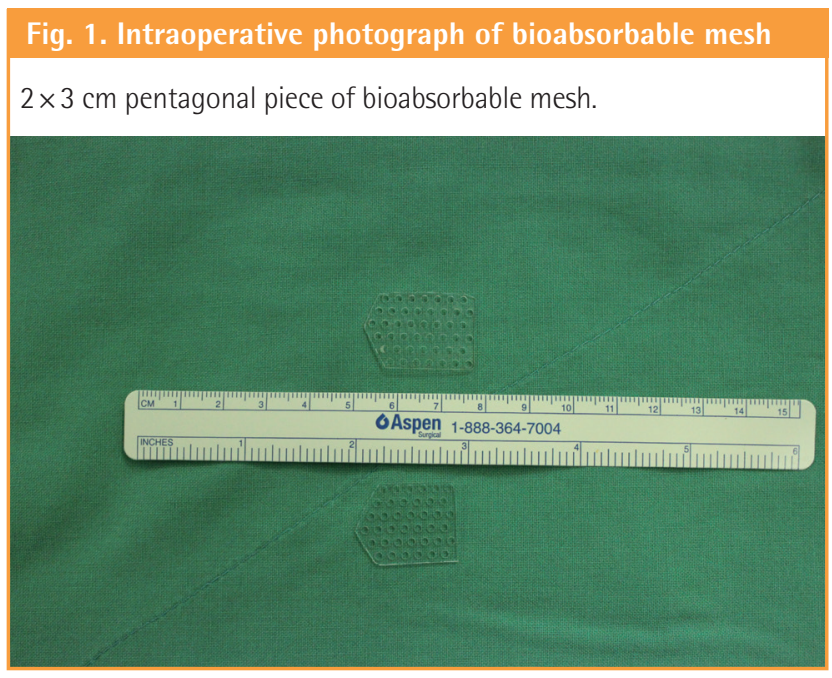

We evaluated the degree of nasal septal dislocation in the coronal plane and of nasal septal displacement in the axial plane by computed tomography. The indication for an operation was nasal septal displacement on CT without dislocation of the nasal septal cartilage from the vomerine groove. Thus, we operated when the cephalic portion of the nasal septal cartilage was comparatively stable. There were no cases of comminuted fracture or septal mucosal laceration. We assessed the patients' symptoms preoperatively by using the nasal obstruction symptom evaluation (NOSE) scale after the swelling subsided, usually on preoperative day 1 (Table 1 ).

The operation was performed 5 to 7 days after the trauma occurred. Every patient was placed under general anesthesia. After reduction of the nasal bone with Asch forceps and a Freer elevator, we approached the septum though an inverted L-shaped incision at the mucocutaneous junction. After elevating the mucoperichondrial flap carefully in order to avoid damage to the nasal mucosa, we inserted a rhinoscope and observed the degree of damage to the nasal septum and the deformed nasal septal cartilage. Dissection was performed up to the perpendicular plate of the ethmoid bone cephalad, up to the vomerine groove caudally, and up to the vomer towards the dorsum; the bent nasal septal cartilage was then corrected by making a cross-hatching incision on the nasal septum and freer elevator. We inserted a $2 \times 3 \mathrm{~cm}$ pentagonal bioabsorbable mesh of $0.75 \mathrm{~mm}$ thickness (Macro-

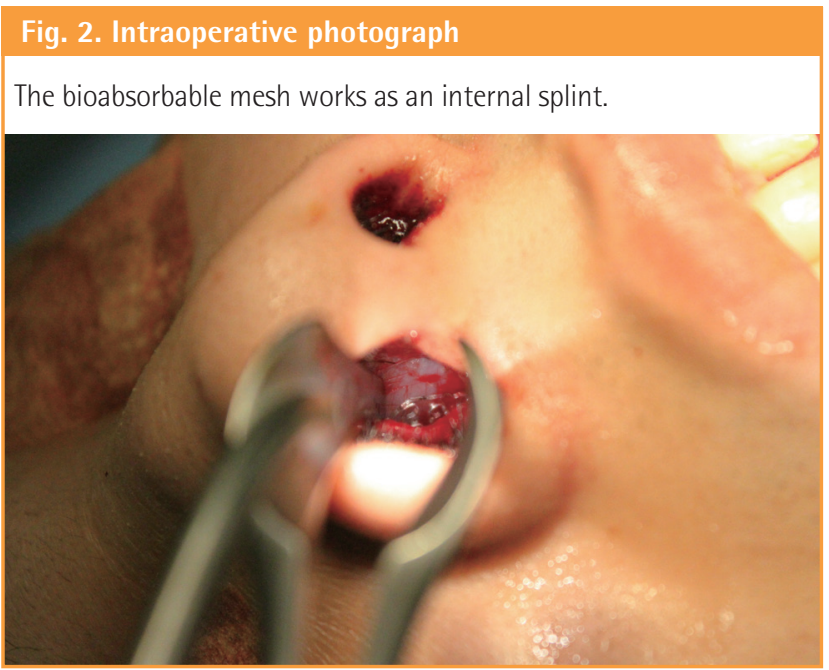

Table 1. Nasal obstruction symptom evaluation scale

\begin{tabular}{|lcccc|}
\hline $\begin{array}{l}\text { How much of a problem were the } \\
\text { following conditions for you? }\end{array}$ & $\begin{array}{c}\text { Not } \\
\text { a problem (0) }\end{array}$ & $\begin{array}{c}\text { Very mild } \\
\text { problem (1) }\end{array}$ & $\begin{array}{c}\text { Moderate } \\
\text { problem (2) }\end{array}$ & $\begin{array}{c}\text { Fairly bad } \\
\text { problem (3) }\end{array}$ \\
\hline $\begin{array}{l}\text { Nasal obstruction } \\
\text { Trouble breathing through my nose }\end{array}$ & & & & \\
Trouble breathing during exercise & & & \\
Trouble sleeping & & & \\
\hline
\end{tabular}


Pore PSTM, Medtronic Inc, San diego, CA, USA) composed of $70 \%$ poly L-lactic acid and $30 \%$ poly D, L-lactic acid into the most deviated part of the nasal septum (Figs. 1, 2) [4]. Considering the possibility of protrusion of mesh in the postoperative period, the bioabsorbable mesh was designed to be smaller than the nasal septal cartilage, and we inserted mesh into the contralateral side only when there was septal mucosal injury during the operation. The incision site was sutured with Vicryl \#5-0 sutures and the sutures were removed five days after the operation. The bioabsorbable mesh was fixed with Vicryl \#4-0 through-andthrough sutures. In order to place the nasal septal cartilage and the bioabsorbable mesh at the desired position, a silicone sheet

\section{Table 2. Patient characteristics}

\begin{tabular}{|c|c|c|c|c|}
\hline \multirow{3}{*}{ Patient } & \multirow{3}{*}{$\begin{array}{l}\text { Age } \\
\text { (yr)/ } \\
\text { Sex }\end{array}$} & \multicolumn{3}{|c|}{$\begin{array}{l}\text { Nasal obstruction symptom } \\
\text { evaluation scale scores }\end{array}$} \\
\hline & & \multirow{2}{*}{ Preoperative } & \multicolumn{2}{|c|}{ Postoperative at } \\
\hline & & & 1-month & 6-month \\
\hline 1 & $24 / M$ & 7 & 3 & 1 \\
\hline 2 & $17 / \mathrm{M}$ & 8 & 4 & 2 \\
\hline 3 & $25 / M$ & 1 & 1 & 1 \\
\hline 4 & $53 / F$ & 9 & 4 & 3 \\
\hline 5 & $23 / F$ & 2 & 1 & 1 \\
\hline 6 & $17 / M$ & 2 & 1 & 1 \\
\hline 7 & $48 / \mathrm{M}$ & 6 & 3 & 1 \\
\hline 8 & $30 / \mathrm{M}$ & 8 & 4 & 1 \\
\hline 9 & $55 / F$ & 1 & 1 & 1 \\
\hline 10 & $32 / \mathrm{M}$ & 12 & 4 & 1 \\
\hline 11 & $27 / M$ & 9 & 4 & 2 \\
\hline 12 & $37 / M$ & 8 & 4 & 1 \\
\hline 13 & $41 / M$ & 7 & 2 & 1 \\
\hline 14 & $26 / F$ & 6 & 1 & 1 \\
\hline 15 & $51 / \mathrm{M}$ & 8 & 3 & 3 \\
\hline 16 & $21 / \mathrm{F}$ & 2 & 1 & 1 \\
\hline 17 & $19 / F$ & 2 & 1 & 1 \\
\hline 18 & $23 / M$ & 5 & 1 & 1 \\
\hline 19 & $24 / M$ & 5 & 2 & 1 \\
\hline 20 & $23 / \mathrm{M}$ & 4 & 1 & 1 \\
\hline
\end{tabular}

was fixed with Vicryl \#4-0 sutures for seven days and the nasal packing was removed five days after the operation. CT and the NOSE scale were repeated at one and six months after the operation, and telephone calls were also made for a period of one to six months after the operation to evaluate patient satisfaction.

Eighteen of twenty patients had symptoms of nasal obstruction before the operation, the average operation time was 86 minutes, and the average patient age was 30.8 years. The range of postoperative follow-up period was 6 to 12 months. A clear improvement was seen in the coronal plane and in the axial plane of CT (Fig. 3). 85\% (17 patients) of the patients were satisfied according to the NOSE scale, and the average postoperative NOSE score was 2.3 at 1 month and 1.3 at 6 months, whereas the average NOSE score before the operation had been 5.6 (Tables 2, 3). Some complications such as septal perforation or infection, redisplacement of the nasal septal cartilage, as well as exposure of the bioabsorbable mesh, were anticipated after the operation, but none of the patients developed any of the anticipated complications during the observation period.

\section{DISCUSSION}

Since nasal bone fractures are the most common among facial bone fractures, they are frequently encountered in clinics. Also, since the operation is comparatively simple, it could be misunderstood to be an easy operation and there is a tendency to overlook the accompanying nasal septal fracture [5]. Kim et

Table 3. Nasal obstruction symptom evaluation (NOSE) scale scores

\begin{tabular}{|lc|}
\hline & NOSE scale scores \\
\hline Preoperative & 5.6 \\
1-month postoperative & 2.3 \\
6-month postoperative & 1.3 \\
\hline
\end{tabular}

\section{Fig. 3. Computed tomography scan}

Case of a 30-year-old man with nasal bone and septal fracture. (A, C) Preoperative computed tomography (CT) scan. (B, D) CT scan taken at 6 months postoperatively.
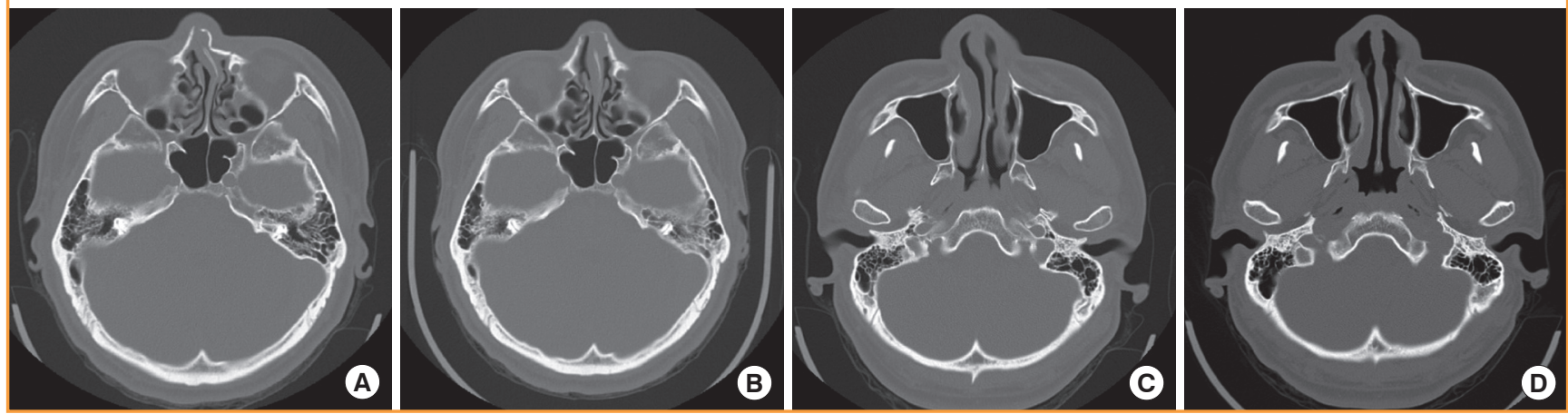
al. [5] reported that $46.9 \%$ of nasal bone fractures are accompanied by septal fractures. Rhee et al. [6] classified nasal septal fractures into four categories according to the degree of damage to the nasal septum seen on CT, and he laid down the principle of performing a septoplasty in cases in which the perpendicular plate of the ethmoid bone and the quadrangular cartilage were dislocated by more than $50 \%$ from the center. However, recently, a septoplasty performed even in cases with a slight dislocation is known to have positive results, and hence the trend is to perform active surgical treatment. Kim et al. [5] achieved a positive result by performing a primary septoplasty in patients with nasal bone fracture accompanied by a small nasal septal fracture.

There is a report that the incidence of nasal deformation ranges from $14 \%$ to $50 \%$ after closed reduction of nasal bone fractures during rhinoplasty or septoplasty [6]. Consequently, the nasal septal damage that may accompany the nasal bone fracture can be the main cause of nasal deformation and nasal obstruction after reduction [2]. Similarly, in cases with a nasal bone fracture accompanied by a traumatic injury to the nasal septal cartilage, there is a more severe secondary nasal deformation than in cases with a nasal bone fracture but without an accompanying nasal septal injury. Hence, the application of an external splint for two weeks after closed reduction is not adequate to prevent secondary deformation of the nasal septal cartilage. Also, the results of septoplasty, which is generally performed with preservation of the nasal septal cartilage, cannot be predicted if there is no adequate clinical experience. Hence, we tried to prevent secondary deformation of the nasal septal cartilage by approaching the nasal septal fracture with open reduction, preserving the nasal septal cartilage, and fixing the bioabsorbable mesh into the perichondrium as an internal splint. This is considered to be a technique that properly complements closed reduction and open reduction performed for nasal septal fractures.

Bioabsorbable mesh is used in craniofacial plastic surgery as well as in facial bone fractures as a treatment option since it causes a negligible foreign body reaction and it does not need to be removed. It can also be used in the patients who are growing because it does not influence growth [4]. We have been using bioabsorbable mesh for years in facial bone fractures and based on this experience, bioabsorbable mesh was easily applied in septoplasty. The bioabsorbable mesh used in this study has a simple design such that it can be easily inserted, does not cause any specific complications, and does not affect the union although it is placed between the septal cartilage and the perichondrium; in addition, the soft tissue can communicate through the various holes located on the mesh [7]. However, since mesh exposure is likely to occur, extra care needs to be taken while elevating and dissecting the perichondrium off the convex side of the septal cartilage, especially the thin flap of perichondrium. The bioabsorbable mesh degrades after two to three years depending on the patient's metabolism. The important point is that the internal splint is absorbed before the bioabsorbable mesh is degraded, and this provides a sufficient time period for the bioabsorbable mesh to function as a buttress after the reduction.

In patients with a nasal bone fracture accompanied by a nasal septal fracture, we reduced the fractured nasal septum while not removing the damaged cartilage during septoplasty. We straightened the bent cartilage by making a cross-hatching incision on the nasal septal cartilage and fixed a piece of bioabsorbable mesh as an internal splint between the perichondrium and the cartilage. Obtaining a satisfactory result in this patient using this technique indicates that open reduction can be easily performed in cases with a nasal septal fracture. However, it is thought that the author's technique requires a longer follow-up, and since the investigation has been performed in only a few patients, this research should be conducted in many cases according to the classification based on the type of nasal septal fractures. There is no data on the interaction between the cartilage and the bioabsorbable mesh, so additional research is needed.

\section{REFERENCES}

1. Ha JS, Lee KC, Kim SK, et al. Classification of nasal bone fracture; types and secondary deformities. J Korean Soc Plast Reconstr Surg 2003;30:287-92.

2. Yoo YS, Jung SH, Yoon ES, et al. Primary septoplasty in the treatment of nasal bone fractures.J Korean Soc Plast Reconstr Surg 2009;36:61-5.

3. Park WY, Kim YH. A clinical study of the nasal bone fracture according to stranc classification. J Korean Soc Plast Reconstr Surg 2008;35:289-94.

4. Losken HW, van Aalst JA, Mooney MP, et al. Biodegradation of Inion fast-absorbing biodegradable plates and screws. J Craniofac Surg 2008;19:748-56.

5. Kim JH, Shin DW, Choi TH, et al. The importance of septoplasty in the treatment of nasal bone and grade I septal fracture: estimation with acoustic rhinometry. J Korean Soc Plast Reconstr Surg 2010;37:626-32.

6. Rhee SC, Kim YK, Cha JH, et al. Septal fracture in simple nasal bone fracture. Plast Reconstr Surg 2004;113:45-52.

7. Shin DH, Kim DJ, Kim SY, et al. Rigid fixation using bioabsorbable mesh and screws in facial bone fracture. J Korean Soc Plast Reconstr Surg 2010;37:717-20. 